\title{
Quantitative correction method for the grinding errors of cycloidal gears in precision reducer
}

\author{
Tianxing LI*, Jinfan LI*, Xiaozhong DENG**, Meng TIAN* and Yulong LI* \\ ${ }^{*}$ School of Mechatronics Engineering, Henan University of Science and Technology, \\ 48 Xiyuan Rd, Luoyang 471003, China \\ E-mail: litianxing@haust.edu.cn \\ ${ }^{* *}$ Collaborative Innovation Center of Machinery Equipment Advanced Manufacturing of Henan Province, \\ 48 Xiyuan Rd, Luoyang 471003, China
}

Received: 10 February 2020; Revised: 26 March 2020; Accepted: 13 April 2020

\begin{abstract}
The motion accuracy of a precision reducer mainly depends on the transmission quality of the cycloid-pin gear, which is determined by the grinding accuracy of the cycloidal gear. The poor meshing characteristics caused by grinding errors in the cycloidal gear are the primary reasons for the low motion accuracy and short service life of a precision reducer. This paper proposes a quantitative correction method based on the grinding error of a cycloidal gear. This method allows the error in machine-tool grinding settings to be quickly and accurately corrected based on mathematical analysis and reverse engineering. A mathematical model of the grinding motion could be constructed based on the particularity of the continuous and closed profile of a cycloidal gear, and a cycloidal profile equation with the grinding settings as variables could be derived. A transitive equation for the grinding settings and tooth profile error was constructed using the closed reconstruction and optimal matching technology, and the essential relationship between the error extraction and quantitative correction was clarified. On this basis, a correction model was constructed, the correction strategy was planned, and the quantified correction of the grinding settings was realized using the numerical optimization method. Experimental results demonstrated that the quantitative correction method could quickly and accurately produce correction values for the machine-tool grinding settings. The tooth profile error could effectively be reduced with just one correction and two grinding operations. This study solved the technical problems that occur in the traditional machining process, which can only be corrected qualitatively and not quantitatively, and provided an effective way to improve the machining precision of the key parts of the precision reducer.
\end{abstract}

Keywords : Precision reducer, Cycloidal gear, Grinding error, Quantitative correction, Machine-tool settings

\section{Introduction}

A precision reducer has a series of advantages compared with a general reducer, including a large transmission ratio, high motion accuracy, strong bearing capacity, and compact structure. As an important part of the mechanical equipment, the transmission performance of the precision reducer directly affects the motion accuracy and repeat positioning accuracy (Wang and Tao, 2014). The precision reducer is mainly composed of an involute cylindrical gear planetary mechanism at the front stage and a cycloid-pin gear planetary mechanism at the rear stage. The cycloid-pin gear mechanism at the low speed end is directly connected to the output shaft. Thus, its transmission performance directly determines the kinematic accuracy of the reducer (Li et al., 2017).

The cycloidal gear is one of the most critical parts of the cycloid-pin mechanism, and its machining quality is the most important factor affecting the meshing characteristics and transmission accuracy (Kawahara et al., 2014; Li et al., 2018). Practical engineering applications have shown that the poor meshing contact characteristics caused by machining error in the cycloidal gear were the main reasons for the low motion accuracy and short service life of a precision reducer. Therefore, controlling the grinding quality of a cycloidal gear is an important prerequisite to ensure that the precision reducer has good motion accuracy. 
The cycloidal gear is a type of special gear with continuous and closed tooth profile. Compared with general gears, the design, processing and assembly of a cycloidal gear are more stringent and difficult. In order to machine the cycloidal gear, Liu et al. (2018) developed a machining method based on the involute shaping cutter and realized the continuous shaping of an effective tooth profile for a compound cycloidal gear. Wang et al. (2018) designed a cycloidal slicing cutter to improve the machining precision and efficiency of a cycloidal gear. Lai (2006) presented a mathematical model to design an epicycloid planet gear for a cycloid drive, and obtained the path for the machining tools of a wire-cutting machine. Chang (2003) obtained the fundamental tooth profile of a hob cutter and the main manufacturing process for the epitrochoid gear, which is beneficial for the manufacture of cycloid drives. Chen et al. (2018) proposed a high-precision technology with negative punch clearance for manufacturing a cycloid pump. With this technology, a long and smooth product surface can be obtained in a few minutes.

Manufacturing error in a cycloidal gear is inevitable. It can cause poor meshing characteristics in the cycloid-pin transmission, and then affect the motion accuracy and service life of the whole machine. Li et al. (2017) concluded that the tooth profile error of the cycloidal gear has greater impacts on the transmission error, and the cycloid-pin transmission at the low-speed stage has a greater influence on the motion accuracy of the whole machine. Li et al. (2018) investigated the influences of the profile modification and eccentricity error on the torsional mesh stiffness, loaded transmission error, Hertzian contact stiffness and load sharing factor. Liu et al. (2019) studied the relationship between the torsional stiffness and surface characteristics of cycloidal gears based on machining parameters. Li et al. (2017) investigated the effects of the radial clearance and pin-hole clearance, as well as eccentricity errors, on the contact stress, transmission error and gear ratio. Trans et al. (2016) concluded that the lost motion of the cycloid reducer depended not only on its torsional stiffness but also on its tolerance. Lin et al. (2018) presented a method for the kinematic error analysis and tolerance allocation of cycloidal gear reducers. Han et al. (2016) studied the influences of the manufacturing errors and assembly errors on the transmission accuracy, and determined the main adjustment errors, which not only improved the transmission accuracy but also reduced the manufacturing cost.

At present, the forming grinding and generating grinding methods are the main methods adopted by manufacturers for actual production. In the process of traditional grinding, in order to obtain the ideal profile, it is necessary for the technicians to estimate the adjustment errors in the grinding settings based on their technical experience or the results of an error inspection, and then repeatedly perform machine-tool adjustments, trial cuts, and error measurements. This tedious process relies solely on the subjective qualitative corrections of the technicians, but cannot quantitatively correct the machine-tool motion, which results in low grinding efficiency, poor profile accuracy, and poor product consistency. In recent years, with the rapid development of gear grinding equipment and precision measurement technology, the machining accuracy inspection of complex surfaces such as bevel gears has been realized, and the technical problems of cycloidal gear measurement have also been overcome (Li et al., 2018). These advances will make the error compensation and correction technology for cycloidal gears more convenient and diverse. Moreover, good engineering results have been achieved using a digital correction method to control and improve the machining accuracy of spiral bevel gears (Li et al., 2019; Wang et al., 2016; Li et al., 2017).

In view of this, the purpose of this study was to develop a method to quantitatively correct the grinding error in a cycloidal gear. Based on the grinding error data of the measured profile, a quantitative correction method for the continuous and closed profile of a cycloidal gear was studied. By establishing the transitive relationship between the tooth profile and the machine-tool grinding settings, a mathematical model was constructed for the quantitative correction of the grinding settings, and the quantitative correction of the grinding error in a cycloidal gear was realized using mathematical analysis and reverse engineering.

\section{Profile equation of cycloidal gear with grinding settings as variables 2.1 Grinding motion analysis of cycloidal gear}

The grinding process for a cycloidal gear can be realized by simulating the planetary transmission of the cycloid-pin gear. During the transmission of the cycloid-pin gear, the pin gear is fixed, and the cycloidal gear makes a counterclockwise revolution around the center of the pin gear. At the same time, this revolution can cause a reaction force on the pin gear, which will lead to a clockwise rotation of the cycloidal gear. When grinding cycloidal gears, a grinding wheel can be used instead of a pin tooth to form a planetary motion relationship with the gear blank installed on the worktable. Figure 1 shows the grinding motion relationship of the cycloidal gear. 


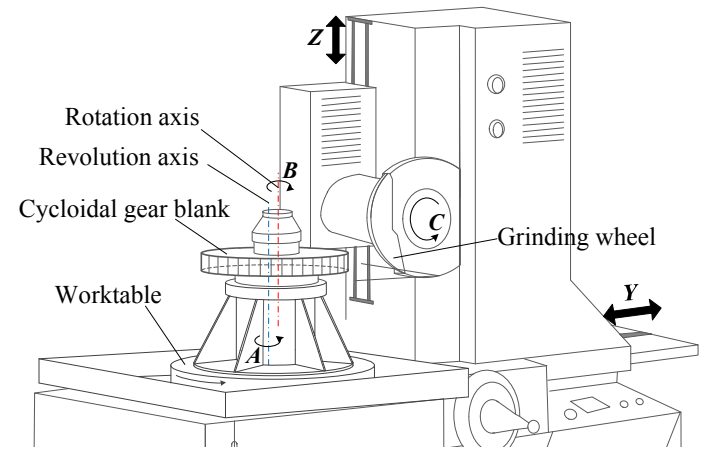

Fig. 1 Grinding motion relationship of cycloidal gear.

During the grinding process, the sliding base of the machine-tool moves along $\mathrm{Y}$ axis to complete the tooth profile processing. The grinding wheel moves up and down along $\mathrm{Z}$ axis to complete the tooth width processing. The machined gear rotates around B axis while simultaneously revolving around A axis to form the planetary motion relationship. The grinding accuracy of the cycloidal profile can be guaranteed by accurately controlling the relative movement trajectories of two linear axes ( $\mathrm{Y}$ and $\mathrm{Z}$ axes) and three rotary axes (A, B and $\mathrm{C}$ axes). Obviously, the rotation and revolution are the most important movements, which are more clearly described in Fig. 2.

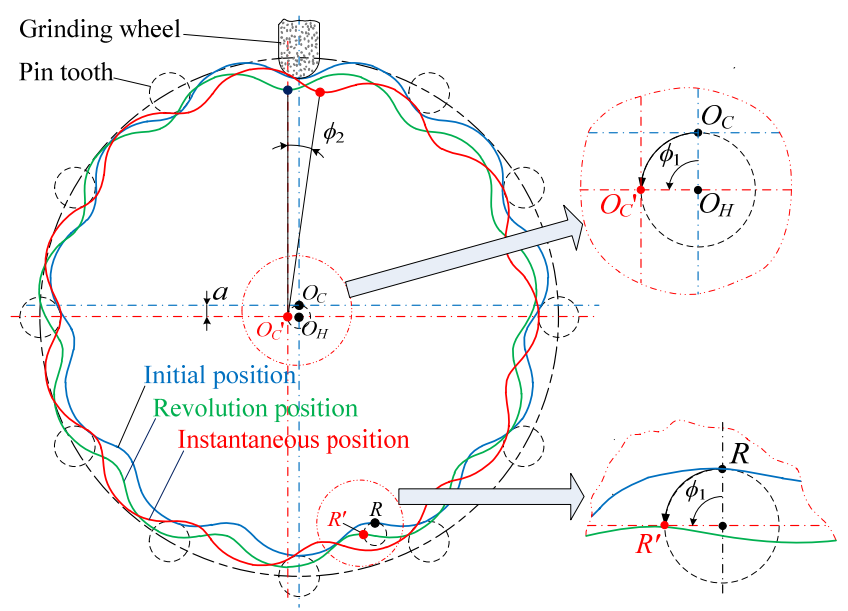

Fig. 2 Revolution and rotation of cycloidal gear.

When the cycloidal gear revolves, center $O_{C}$ of the cycloidal gear rotates around center $O_{H}$ of the pin gear. Assuming that the revolution angle of A axis is $\phi_{1}$, the cycloidal gear is stationary relative to its own axis, and the gear blank rotates by angle $\phi_{1}$ around $O_{H}$. At this time, center $O_{C}$ of the cycloidal gear moves to $O_{C^{\prime}}$, and point $R$ on the cycloidal profile moves to point $R^{\prime}$, which is shown as a green solid line in Fig. 2. On this basis, when the cycloidal gear rotates, any point on the cycloidal profile rotates around its own axis. In other words, there is rotational motion around central axis $B$. The red solid line in Fig. 2 shows the instantaneous positions of the cycloidal gear at rotation angle $\phi_{2}$. When the rotating speed ratio of A and B axes meets the grinding conditions, the gear blank and grinding wheel are in the correct grinding contact state, and the grinding of the tooth profile can be completed.

\subsection{Grinding model and profile equation of cycloidal gear}

The cycloidal gear profile equation has been derived by many researchers (Litvin and Feng, 1996; Chang,2003; Lin et al., 2018). However, the geometric parameters of the cycloid-pin gear are taken as variables in these profile equations. In order to effectively correct the grinding error, it is necessary to associate the tooth profile equation of the cycloidal gear with the grinding setting parameters of the machining equipment. In this paper, a mathematical model for the grinding can be constructed based on the grinding motion relationship, as shown in Fig. 3. $S_{H}\left(X_{H}, Y_{H}, Z_{H}\right)$ is a fixed coordinate system, which is connected to the machine-tool frame. Coordinate system $S_{G}\left(X_{G}, Y_{G}, Z_{G}\right)$ is connected to the grinding wheel, where axis $X_{G}$ is rotation axis $C$ of the grinding wheel. Coordinate system $S_{1}\left(X_{1}, Y_{1}, Z_{1}\right)$ is an 
auxiliary coordinate system, which represents the revolution position of the machined gear blank. Origin $O_{1}$ is determined by rotation angle $\phi_{1}$ and eccentricity $a$. Coordinate system $S_{C}\left(X_{C}, Y_{C}, Z_{C}\right)$ is firmly connected to the cycloidal gear and is obtained by rotating coordinate system $S_{1}$ around axis $Z_{1}$ by angle $\phi_{2}$. $E$ and $L$ determine the position of grinding wheel center $O_{G}$ with respect to revolution center $O_{H}{ }^{\prime}$ of the cycloidal gear in plane $Y_{H} Z_{H}$. $H$ is the Z-direction distance of revolution center $O_{H}{ }^{\prime}$ of the cycloidal gear in $S_{H}$. In addition, $r_{g}$ is the nominal radius of the grinding wheel and $r_{r p}$ is the arc radius of the grinding blade.

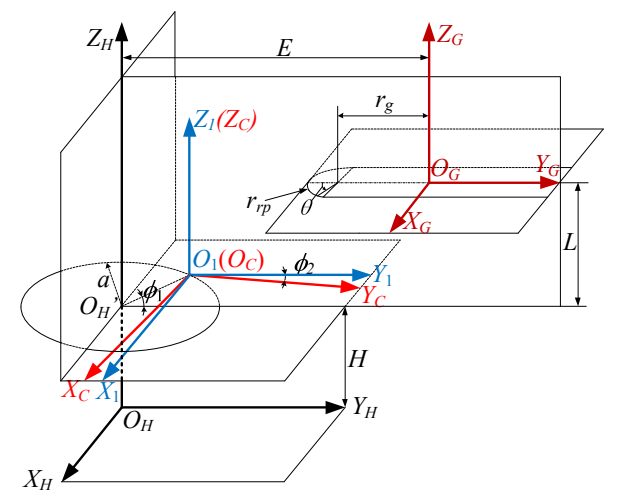

Fig. 3 Grinding model of cycloidal gear.

According to the grinding model, the main machine-tool grinding settings include speed ratio $i$, eccentricity $a$, nominal radius $r_{g}$ of the grinding wheel, arc radius $r_{r p}$ of the grinding blade, radial feed distance $E$ and vertical feed distance $L$. In this paper, $\boldsymbol{\Phi}_{j}=\left\{i, r_{r p}, a, r_{g}, E, L\right\}$ (where $j=1,2, \ldots$ indicates the number of grinding settings) is introduced to represent the set of machine-tool grinding settings. According to the gear meshing principle and differential geometry theory, the tooth profile equation of the cycloidal gear can be derived through the conversion of the conjugate position at any point on the grinding blade.

The position vector and unit normal vector of the grinding blade in $S_{G}$ can be expressed as follows:

$$
\begin{aligned}
& \mathbf{R}_{G}\left(\theta ; \boldsymbol{\Phi}_{j}\right)=\left[r_{r p} \sin \theta,-r_{g}-r_{r p} \cos \theta, 0,1\right]^{T} \\
& \mathbf{n}_{G}\left(\theta ; \boldsymbol{\Phi}_{j}\right)=[-\sin \theta, \cos \theta, 0]^{T}
\end{aligned}
$$

where $\theta \in[-\pi / 2, \pi 2]$ is measured in the negative direction of $Y_{G}$ axis. Note that Eq. (1) is a three-dimensional position vector, which is represented as a homogeneous vector for coordinate transformation. "1" in the last element of Eq. (1) is only for the homogeneous transformation matrix in Eq. (4).

Using the space coordinate transformation technology (Litvin and Feng, 1996; Lin et al., 2018), the position vector and unit normal vector of the cycloidal gear in $S_{C}$ can be derived as follows:

$$
\left\{\begin{array}{l}
\mathbf{R}_{C}\left(\theta, \phi_{2} ; \boldsymbol{\Phi}_{j}\right)=\mathbf{M}_{C G} \mathbf{R}_{G}\left(\theta ; \boldsymbol{\Phi}_{j}\right) \\
\mathbf{n}_{C}\left(\theta, \phi_{2} ; \boldsymbol{\Phi}_{j}\right)=\mathbf{L}_{C G} \mathbf{n}_{G}\left(\theta ; \boldsymbol{\Phi}_{j}\right) \\
x_{C}{ }^{\prime}(\theta) y_{C}{ }^{\prime}\left(\phi_{2}\right)-x_{C}{ }^{\prime}\left(\phi_{2}\right) y_{C}{ }^{\prime}(\theta)=0
\end{array}\right.
$$

where $\partial \mathbf{R}_{C} / \partial \theta=\left[\begin{array}{ll}x_{C}{ }^{\prime}(\theta) & y_{C}{ }^{\prime}(\theta)\end{array}\right]^{T}, \quad \partial \mathbf{R}_{C} / \partial \phi_{2}=\left[\begin{array}{ll}x_{C}{ }^{\prime}\left(\phi_{2}\right) & y_{C}{ }^{\prime}\left(\phi_{2}\right)\end{array}\right]^{T}$, and $\mathbf{M}_{C G}$ is the transformation matrix from $S_{G}$ to $S_{C} . \mathbf{L}_{C G}$ is obtained by removing the last row and last column from $\mathbf{M}_{C G}$.

$$
\mathbf{M}_{C G}=\left[\begin{array}{cccc}
\cos \phi_{2} & -\sin \phi_{2} & 0 & a \sin \left(\phi_{1}+\phi_{2}\right)-E \sin \phi_{2} \\
\sin \phi_{2} & \cos \phi_{2} & 0 & -a \cos \left(\phi_{1}+\phi_{2}\right)+E \cos \phi_{2} \\
0 & 0 & 1 & L \\
0 & 0 & 0 & 1
\end{array}\right]
$$


The theoretical tooth profile equation and unit normal vector of the cycloidal gear in $S_{C}$ can be obtained by simplifying Eq. (3).

$$
\begin{array}{r}
\mathbf{R}_{C}\left(\phi_{2} ; \boldsymbol{\Phi}_{j}\right)=\left[\begin{array}{r}
r_{r p} \sin \left(\arctan \left(\left(a(i+1) \sin \left(i \phi_{2}\right)\right) /\left(a(i+1) \cos \left(i \phi_{2}\right)-\left(E-r_{g}\right)\right)\right)+\phi_{2}\right) \\
-\left(E-r_{g}\right) \sin \phi_{2}+a \sin \left((i+1) \phi_{2}\right) \\
-r_{r p} \cos \left(\arctan \left(\left(a(i+1) \sin \left(i \phi_{2}\right)\right) /\left(a(i+1) \cos \left(i \phi_{2}\right)-\left(E-r_{g}\right)\right)\right)+\phi_{2}\right) \\
+\left(E-r_{g}\right) \cos \phi_{2}-a \cos \left((i+1) \phi_{2}\right)
\end{array}\right] \\
\mathbf{n}_{C}\left(\phi_{2} ; \boldsymbol{\Phi}_{j}\right)=\left[\begin{array}{r}
-\sin \left(\arctan \left(\left(a(i+1) \sin \left(i \phi_{2}\right)\right) /\left(a(i+1) \cos \left(i \phi_{2}\right)-\left(E-r_{g}\right)\right)\right)+\phi_{2}\right) \\
\cos \left(\arctan \left(\left(a(i+1) \sin \left(i \phi_{2}\right)\right) /\left(a(i+1) \cos \left(i \phi_{2}\right)-\left(E-r_{g}\right)\right)\right)+\phi_{2}\right)
\end{array}\right]
\end{array}
$$

It can be seen from Eqs. (5) and (6) that the tooth profile geometry of the cycloidal gear is mainly determined by the machine-tool grinding settings, $\boldsymbol{\Phi}_{j}$. When the grinding motion relationships are set without errors, the actual and theoretical profiles are completely coincident, and the grinding error is zero.

\section{Transitive relationship between grinding settings and profile errors}

After grinding, the tooth profile error, pitch error and radial runout error of the cycloidal gear can be obtained based on the gear measuring center ( $\mathrm{Li}$ et al., 2018). Among these errors, the pitch and radial runout errors are mainly dependent on the inherent accuracy of the grinding equipment, and the tooth profile error is mainly determined by the adjustment error of the machine-tool grinding settings, $\boldsymbol{\Phi}_{j}$. The profile error of the cycloidal gear refers to the deviation of the actual tooth profile from the theoretically designed profile, which is measured along the normal direction of the theoretical profile. Based on the discrete error data obtained from the gear measuring center, the closed reconstruction and optimal matching of the continuous profile of the cycloidal gear are the keys to accurately correcting the grinding error.

Because of the existence of grinding error, the actual profile of the cycloidal gear is no longer a standardized curve, but a free curve. Therefore, the actual profile cannot be simply represented by an elementary curve. In this paper, the cubic B-spline curve is used to reconstruct and express the actual tooth profile corresponding to the discrete measured points of the cycloidal gear. An actual tooth profile equation containing the grinding error information can be indirectly obtained by calculating the knot vectors and inverse of the control vertices. It can be expressed as follows:

$$
\mathbf{H}_{C}(u)=\sum_{i=0}^{m} N_{i, 3}(u) \mathbf{D}_{i}
$$

where $\left\{\mathbf{D}_{i}\right\}$ represents the control vertices, which are calculated based on the discrete points or machining errors. In addition, $m$ is the number of node points on the discrete profile, and $u$ is the surface parameter, $0 \leq u \leq 1$. Here, $\left\{N_{i, 3}(u)\right\}$ are the 3 rd degree B-spline basis functions defined on the nonuniform knot vector $\mathbf{U}=\left\{0,0,0,0, u_{4}, \cdots, u_{m}, 1,1,1,1\right\}$.

In addition, because of the influences of the starting point error, installation error, and instrument system error during the measurement process, the initial discrete error obtained after the profile measurement cannot truly reflect the processing quality of the actual tooth profile. There is an incorrect relative position relationship between the actual measured profile and the theoretically designed profile, as shown in Fig. 4. In the figure, the deviation angle, $\gamma$, represents the inconsistency between the theoretical and actual starting measurement points. $\Delta x$ and $\Delta y$ are the translational deviations, which are installation errors caused by eccentricity errors, instrument system errors, etc. Obviously, the result of the correction in this case will be insufficient for actual production. It is necessary to produce an optimal match between the theoretical profile and the actual profile. 


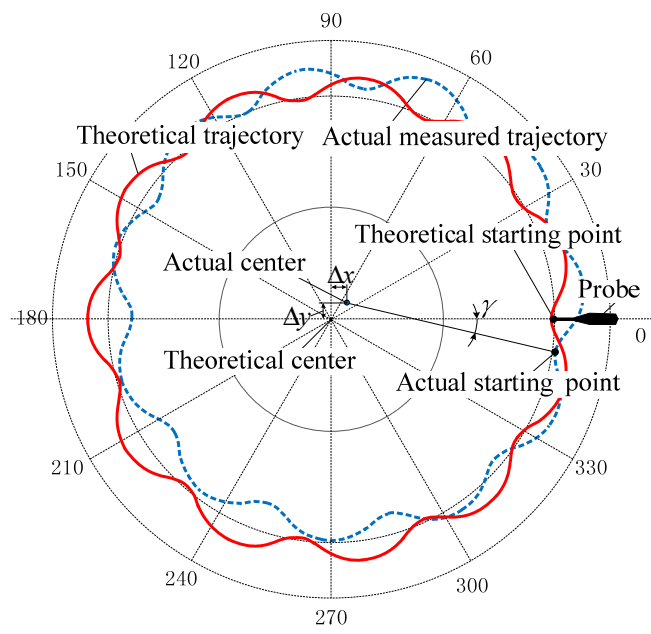

Fig. 4 Deviation of initial measurement point between theoretical and actual profiles.

In order to reduce or eliminated the influences of the starting point error, installation error, and instrument system error, an optimal posture matching method is described in this paper. The least square sum of the errors of each measured point is used as the objective function. The translation and rotation of each coordinate axis are design variables, and the comprehensive effects of the degree of freedom of each axis on the relative positions of the theoretical and actual profiles are taken as the constraints. Assuming that $P_{i}$ (where $i=1,2, \ldots, n, n$ is the number of measured points) is a point on the theoretical profile $\mathbf{R}_{C}$ of the cycloidal gear, the optimal matching goal is to determine a rigid matrix, $\mathbf{M}$, so that the least square sum of the distance from a theoretical point along the normal direction to the actual profile is the minimum.

$$
F_{\min }(\Delta x, \Delta y, \gamma)=\sum_{i=1}^{n} D^{2}\left(P_{i}, \mathbf{H}_{C}{ }^{*}(u)\right)=\sum_{i=1}^{n} D^{2}\left(P_{i}, \mathbf{M}(\Delta x, \Delta y, \gamma) \cdot \mathbf{H}_{C}(u)\right)
$$

where $\mathbf{H}_{C}{ }^{*}(u)$ is the transformed profile from $\mathbf{H}_{C}(u)$; and $\gamma, \Delta x$, and $\Delta y$ represent the rotation angle and translation distances of $\mathbf{H}_{C}(u)$ around axes $X_{c}$ and $Y_{c}$ in $S_{c}$, respectively. $\mathbf{M}=\left[\begin{array}{cccc}\cos \gamma & \sin \gamma & 0 & 0 \\ -\sin \gamma & \cos \gamma & 0 & 0 \\ 0 & 0 & 1 & 0 \\ 0 & 0 & 0 & 1\end{array}\right]\left[\begin{array}{cccc}1 & 0 & 0 & \Delta x \\ 0 & 1 & 0 & \Delta y \\ 0 & 0 & 1 & 0 \\ 0 & 0 & 0 & 1\end{array}\right]$ is the transformation matrix.

The optimal values of $\gamma, \Delta x$ and $\Delta y$ can be optimized using the numerical iterative method. Then, the actual machined profile, $\mathbf{H}_{C}{ }^{*}(u)$, has the correct relative position relationship with the theoretical profile, $\mathbf{R}_{C}\left(\phi_{2} ; \boldsymbol{\Phi}_{j}\right)$, which can be expressed as follows:

$$
\mathbf{H}_{C}^{*}(u)=\mathbf{R}_{C}\left(\phi_{2} ; \boldsymbol{\Phi}_{j}\right)+\delta\left(\phi_{2} ; \boldsymbol{\Phi}_{j}\right) \cdot \mathbf{n}_{C}\left(\phi_{2} ; \boldsymbol{\Phi}_{j}\right)
$$

where $\delta$ is the grinding error of the cycloidal gear. It should be noted that each point on the theoretical tooth profile $\mathbf{R}_{C}$ corresponds to an error $\delta$, and the position vector of this point is determined by the grinding settings, $\boldsymbol{\Phi}_{j}$, and the parameter, $\phi_{2}$. Therefore, the error $\delta$ can be considered as a function determined by $\boldsymbol{\Phi}_{j}$ and $\phi_{2}$.

Equation (9) represents the transitive relationship between the grinding settings, $\boldsymbol{\Phi}_{j}$, and the tooth profile error, $\delta$, and it also reflects the reversible process of error extraction and quantitative correction. When correcting grinding errors, $\mathbf{H}_{C}{ }^{*}$ can be determined using Eq. (8). $\mathbf{R}_{C}, \mathbf{n}_{C}$ and $\delta$ depend on $\boldsymbol{\Phi}_{j}$ and $\phi_{2}$. Thus, Eq. (9) can be regarded as a nonlinear system of equations with $\boldsymbol{\Phi}_{j}$ and $\phi_{2}$ as optimization variables. The optimal grinding settings, $\boldsymbol{\Phi}_{j}{ }^{*}$, can be optimized to make $\delta$ tend to be minimal. 


\section{Quantitative correction method for grinding error}

The essence of the traditional correction method is to continuously change the actual grinding settings to make the actual machined profile gradually approach the theoretical profile. When the deviation between the two profiles is within a certain tolerance range, the correction results can be considered valid. Each adjustment of the grinding movement is qualitative rather than quantitative and accurate, which is also the main reason for the low machining efficiency and poor grinding quality.

In order to solve this technical problem, this paper proposes a new correction method, which is the opposite of the traditional adjustment sequence. It is known that the theoretical profile corresponds to the theoretical grinding settings, and the actual profile also corresponds to the actual grinding settings. Based on this consideration, by changing the theoretical grinding settings, the theoretically designed profile gradually gets closer to the actual profile until they are fully consistent. At this time, the corrected grinding settings can be considered to be the actual machine-tool settings corresponding to the machined profile. The difference between the actual and theoretical grinding settings is the compensation correction value for the re-grinding process. The specific correction principle is shown in Fig. 5 .

In Fig. 5, $k$ is introduced to represent the number of corrections, and $\Delta \boldsymbol{\Phi}_{j}^{(k)}=\left(\Delta i^{(k)}, \Delta r_{r p}^{(k)}, \Delta a^{(k)}, \Delta r_{g}^{(k)}, \Delta E^{(k)}, \Delta L^{(k)}\right)$ represents the k-th corrections of the grinding settings. $\boldsymbol{\Phi}_{j}^{(0)}$ represents the initial or theoretical grinding settings, which correspond to the theoretically designed profile $\mathbf{R}_{C} \cdot \boldsymbol{\Phi}_{j}^{(k)}=\boldsymbol{\Phi}_{j}^{(0)}+\Delta \boldsymbol{\Phi}_{j}^{(k)}$ represents the k-th grinding settings. The deviation between $\mathbf{R}_{C}$ and $\mathbf{H}_{C}{ }^{*}$ is recorded as $\delta$. Given a smaller correction, $\Delta \boldsymbol{\Phi}_{j}^{(1)}$, the corresponding corrected profile $\mathbf{R}_{C}^{(1)}$ can be obtained. The error between $\mathbf{R}_{C}^{(1)}$ and $\mathbf{H}_{C}{ }^{*}$ is $\delta^{(1)}$, and $\delta^{(1)}<\delta$. When correction $\Delta \boldsymbol{\Phi}_{j}^{(2)}$ is further increased, corrected profile $\mathbf{R}_{C}^{(2)}$ again moves closer to $\mathbf{H}_{C}{ }^{*}$, and $\delta^{(2)}<\delta^{(1)}$. In this way, correction $\Delta \boldsymbol{\Phi}_{j}^{(k)}$ is continuously increased until the error, $\delta^{(k)}$, between corrected profile $\mathbf{R}_{C}^{(k)}$ and actual profile $\mathbf{H}_{C}{ }^{*}$ is minimized. At this time, the grinding settings of the corrected profile can be obtained as $\boldsymbol{\Phi}_{j}^{*}=\boldsymbol{\Phi}_{j}^{(0)}+\Delta \boldsymbol{\Phi}_{j}^{(k)}$, and the optimal correction is $\Delta \boldsymbol{\Phi}_{j}^{*}=\Delta \boldsymbol{\Phi}_{j}^{(k)}$. Because the corrected profile is infinitely close to the actual profile, the grinding settings of the actual profile can be approximately considered to be $\boldsymbol{\Phi}_{j}^{*}$.

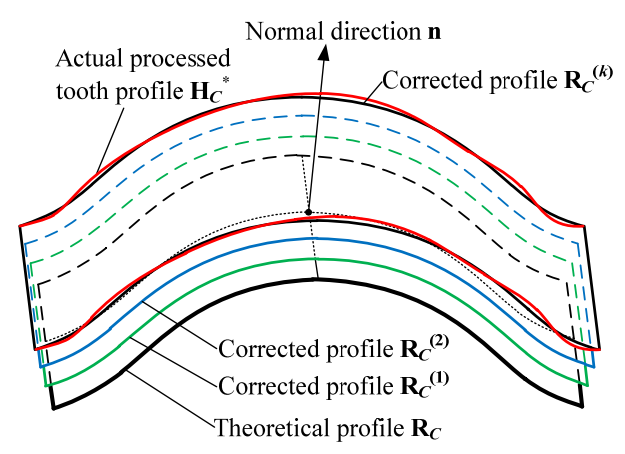

Fig. 5 Process and strategy for quantitative correction.

During the correction process, the actual tooth profile, $\mathbf{H}_{C}{ }^{*}$, remains unchanged, and different grinding settings, $\mathbf{\Phi}_{j}^{(k)}=\left(i^{(k)}, r_{r p}^{(k)}, a^{(k)}, r_{g}^{(k)}, E^{(k)}, L^{(k)}\right)$, correspond to different correction profiles, $\mathbf{R}_{C}^{(k)}\left(\phi_{2} ; \boldsymbol{\Phi}_{j}^{(k)}\right)$. Therefore, Eq. (9) can be written as follows:

$$
\boldsymbol{H}_{C}{ }^{*}\left(u_{i}\right)=\mathbf{R}_{C}^{(k)}\left(\phi_{2 i} ; \boldsymbol{\Phi}_{j}^{(k)}\right)+\delta_{i}^{(k)}\left(\phi_{2 i} ; \boldsymbol{\Phi}_{j}^{(k)}\right) \cdot \mathbf{n}_{C}^{(k)}\left(\phi_{2 i} ; \boldsymbol{\Phi}_{j}^{(k)}\right)
$$

where superscript $k$ indicates the number of corrections and subscripts $i=1-n$ (where $n$ is the number of measured points) represent a series of discrete measured points on the actual profile.

Therefore, $\Delta \boldsymbol{\Phi}_{j}^{(k)}$ and $\phi_{2 i}$ can be used as design variables to solve the optimal grinding settings, $\boldsymbol{\Phi}_{j}^{(*)}$, to minimize the square sum of the errors. The constructed optimization model can be expressed as follows: 


$$
\min f\left(\phi_{2 i} ; \Delta \boldsymbol{\Phi}_{j}^{(k)}\right)=\min \sum_{i=1}^{n}\left|\delta_{i}^{(k)}\left(\phi_{2 i} ; \boldsymbol{\Phi}_{j}^{(k)}\right)\right|^{2}=\min \sum_{i=1}^{n}\left|\left(\mathbf{H}_{C}{ }^{*}\left(u_{i}\right)-\mathbf{R}_{C}^{(k)}\left(\phi_{2 i} ; \boldsymbol{\Phi}_{j}^{(k)}\right)\right) \cdot \mathbf{n}_{C}^{(k)}\left(\phi_{2 i} ; \boldsymbol{\Phi}_{j}^{(k)}\right)\right|^{2}
$$

where $n$ is the number of measured points on the actual profile.

Because the factors influencing the geometric accuracy are not limited to the machine-tool grinding settings, the corrected profile and actually machined profile cannot completely coincide. Thus, Eq. (11) is a nonlinear optimization problem. The optimal grinding corrections, $\Delta \boldsymbol{\Phi}_{j}^{(*)}$, can be determined using an iterative optimization algorithm. When grinding again, the new settings, $\boldsymbol{\Phi}_{j}^{(\text {new })}$, should be written as follows:

$$
\boldsymbol{\Phi}_{j}^{(n e w)}=\boldsymbol{\Phi}_{j}-\Delta \boldsymbol{\Phi}_{j}^{(*)}
$$

The flow for the quantitative correction of the grinding error of the cycloidal gear is shown in Fig. 6 .

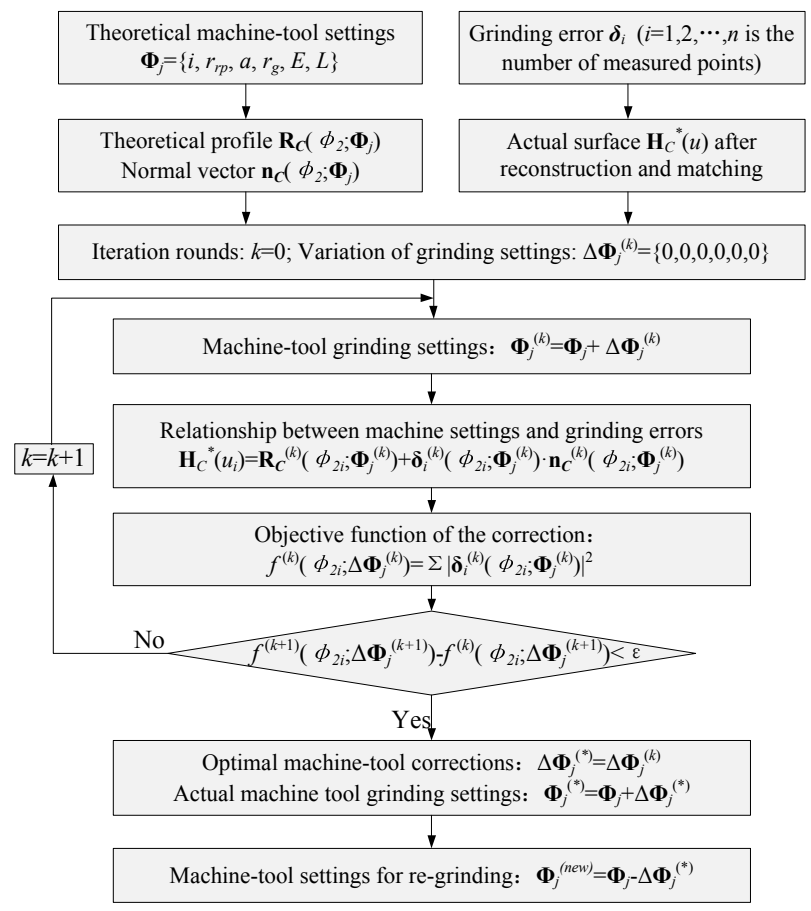

Fig. 6 Flow chart of quantitative correction.

\section{Examples and discussion}

In this study, the process of grinding, measuring, and correcting the cycloidal gear of a robot reducer was taken as an example. The experimental equipment included a YK7350B CNC gear grinding machine and JD45+ gear measuring center, as shown in Figs. 7 and 8, respectively. The YK7350B grinding machine is a modern CNC gear manufacturing device. The maximum diameter that it can process is $500 \mathrm{~mm}$, the maximum tooth width that it can process is $150 \mathrm{~mm}$, the indexing accuracy reaches 1 arcmin, and the precision grade of the processed gear is up to 4 , which met the requirements of the grinding experiment. The JD45+ gear measuring center uses a fully closed-loop digital AC servo control system and an inductive probe system with protective functions. The measurement uncertainties of the helix and involute are $1.5 \mu \mathrm{m}$ and $1.0 \mu \mathrm{m}$, respectively (the coverage factor was 2 ). The total deviation repeatability under the same measuring conditions is less than or equal to $0.5 \mu \mathrm{m}$. A probe with a diameter of $3.0 \mathrm{~mm}$ was selected, and its ball roundness is $1 / 500 \mathrm{~mm}$, which met the requirement of the measurement experiment. 
Tianxing Li, Jinfan Li, Deng, Tian and Yulong Li,

Journal of Advanced Mechanical Design, Systems, and Manufacturing, Vol.14, No.4 (2020)

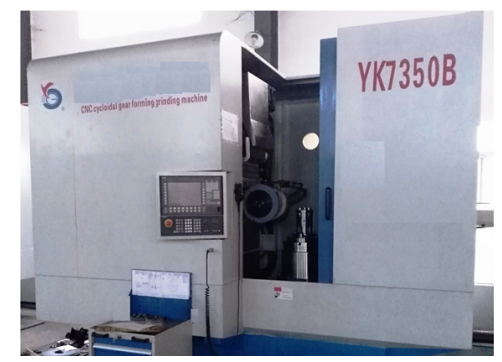

Fig. 7 YK7350B CNC gear grinding machine.

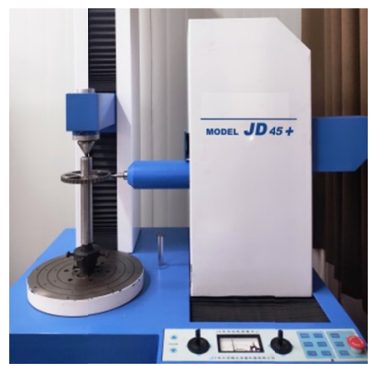

Fig. 8 JD45+ gear measuring center.

The basic parameters and grinding settings of the cycloidal gear are listed in Tables 1 and 2, respectively.

Table 1 Basic parameters of cycloid-pin gear.

\begin{tabular}{lc}
\hline \multicolumn{1}{c}{ Parameter } & Value \\
\hline Number of teeth in cycloidal gear & 39 \\
Number of teeth in pin gear & 40 \\
Roller radius & $3.0 \mathrm{~mm}$ \\
Eccentricity & $1.3 \mathrm{~mm}$ \\
Roller position & $64 \mathrm{~mm}$ \\
\hline
\end{tabular}

Table 2 Theoretical machine-tool grinding settings.

\begin{tabular}{lc}
\hline \multicolumn{1}{c}{ Machine-tool grinding setting } & Value \\
\hline Speed ratio $i$ & 39 \\
Arc radius $r_{r p}$ of the grinding blade & $3 \mathrm{~mm}$ \\
Eccentricity $a$ & $1.3 \mathrm{~mm}$ \\
Nominal radius $r_{g}$ of grinding wheel & $100 \mathrm{~mm}$ \\
Radial feed distance $E$ & $164 \mathrm{~mm}$ \\
Vertical feed distance $L$ & $10 \mathrm{~mm}$ \\
\hline
\end{tabular}

Based on the grinding model of the cycloidal gear, angle $\phi_{2}$ was discretely divided into 3600 parts and used in Eqs. (5) and (6). The coordinates and normal vector of the discrete points on the theoretical profile in $S_{C}$ could be calculated. These data were imported into the $\mathrm{CNC}$ grinding machine to adjust and dress the grinding wheel. The first grinding process for the cycloidal gear was completed, as shown in Fig. 9. 


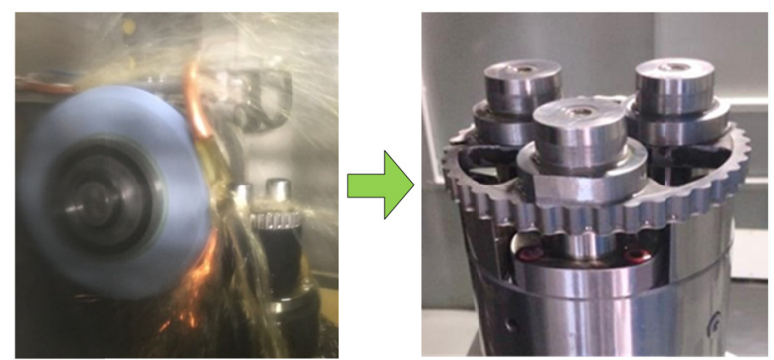

Fig. 9 First grinding process for cycloidal gear.

After the first grinding process, the cycloidal gear was installed on the JD45+ gear measuring center. With the help of the cycloidal gear manufacturing error measurement software developed by the research group, the tooth root point was used as the theoretical initial measurement point. Based on the calculated theoretical profile data, the computer controlled each axis to complete the inspection of all the teeth of the cycloidal gear, as shown in Fig. 10.

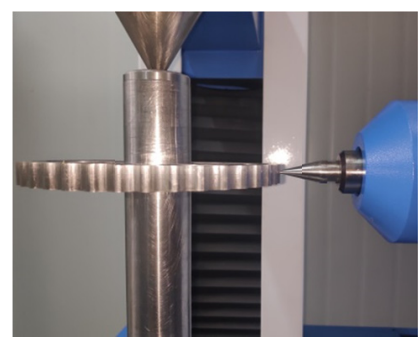

Fig. 10 Tooth profile measurement of cycloidal gear.

The actual coordinates of the cycloidal gear were obtained after the measurement. The relative positions of the theoretical and measured profiles are drawn in Fig. 11. Points $A$ and $A^{*}$ are the tooth root points of the theoretical and actual profiles, respectively. They should also be the theoretical and actual starting measurement points. However, the actual measurement point was not point $A^{*}$, but point $B$. Therefore, there was a deviation angle, $\gamma$, between the theoretical and actual profiles. In addition, due to the influence of installation errors, the actual center of the gear blank deviates from the theoretical center, and its coordinates are $(\Delta x, \Delta y)$.

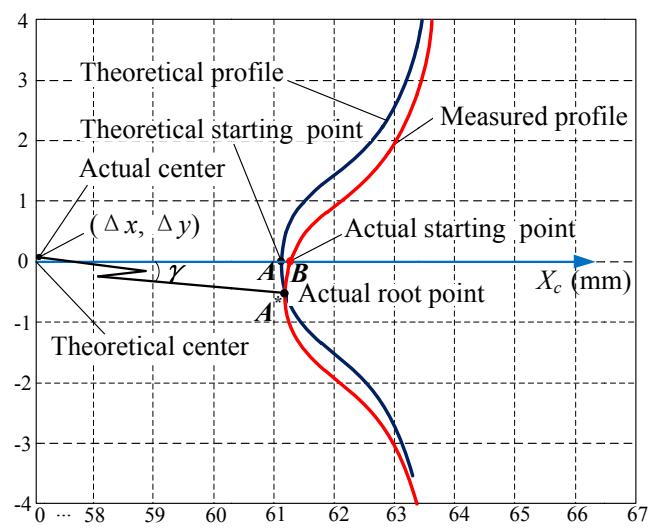

Fig. 11 Initial relative positions of theoretical and measured profiles.

In order to reduce or eliminate the influences of the starting measurement point and the installation error, the degrees of freedom $\gamma, \Delta x$ and $\Delta y$ for each axis were optimized as design variables using the simplex method. The optimal results were $\gamma=8.339 \times 10^{-3} \mathrm{rad}, \Delta x=-2.543 \times 10^{-5} \mathrm{~mm}$, and $\Delta y=-1.119 \times 10^{-5} \mathrm{~mm}$. The corresponding objective function value was reduced from $8.516 \mathrm{~mm}^{2}$ before the matching to $0.027 \mathrm{~mm}^{2}$. 
According to Eq. (9), the tooth profile error distribution and comprehensive error trend after the first grinding were obtained using the Newton-Raphson optimization method, as shown in Figs. 12 and 13, respectively. These results reflect the deviation of the theoretical and actual profiles of the cycloidal gear in the normal direction, and they are also a true reflection of the error of the machine-tool grinding settings.

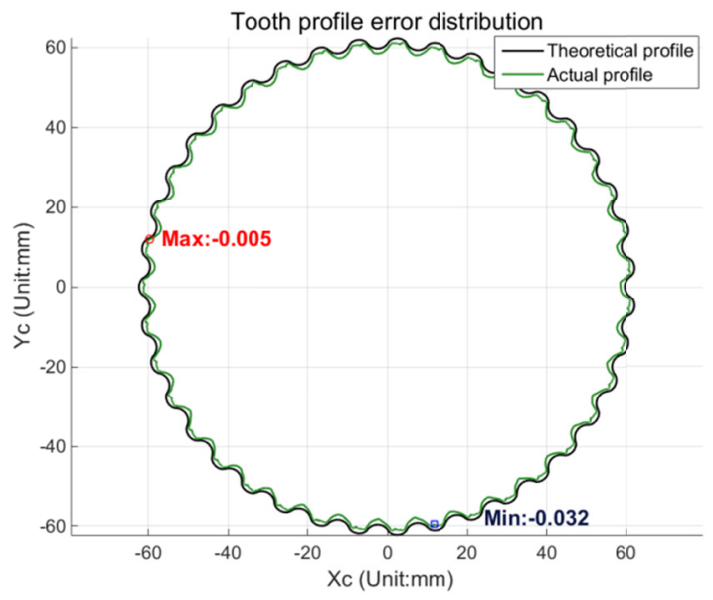

Fig. 12 Tooth profile error distribution after first grinding.

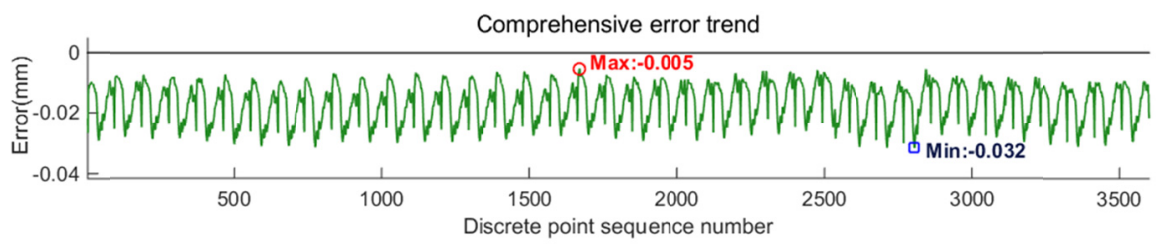

Fig. 13 Comprehensive error trend of cycloidal gear after first grinding.

It can be seen from the measurement results in Figs. 12 and 13 that the overall error trend of the cycloidal gear is negative. The maximum error was $-0.005 \mathrm{~mm}$, and the minimum error was $-0.032 \mathrm{~mm}$. The total error fluctuation range reached $0.027 \mathrm{~mm}$. It could preliminarily be determined that during the actual grinding, the radial feed distance of the grinding wheel was too small, which resulted in a small geometric size for the gear blank. It was necessary to perform a compensation correction of the grinding settings. According to Eqs. (9) and (11), the quantitative correction of the grinding settings was completed using Newton-Raphson optimization method, as listed in Table 3.

Table 3 Optimal corrections of grinding settings.

\begin{tabular}{lcc}
\hline \multicolumn{1}{c}{ Machine-tool setting } & Correction & Actual grinding setting \\
\hline Speed ratio $i$ & 0 & 39 \\
Arc radius $r_{r p}$ of the grinding blade & $-0.012 \mathrm{~mm}$ & $3.012 \mathrm{~mm}$ \\
Eccentricity $a$ & $-0.001 \mathrm{~mm}$ & $1.301 \mathrm{~mm}$ \\
Nominal radius $r_{g}$ of grinding wheel & $0 \mathrm{~mm}$ & $100 \mathrm{~mm}$ \\
Radial feed $E$ & $-0.035 \mathrm{~mm}$ & $164.035 \mathrm{~mm}$ \\
Vertical feed $L$ & $0 \mathrm{~mm}$ & $10 \mathrm{~mm}$ \\
\hline
\end{tabular}

By superimposing the corrections on the theoretical grinding settings, the actual grinding settings could be determined (Table 3), and a corrected profile that was sufficiently close to the actual profile could also be obtained. In order to compare the correction effect, the average error change trend of the single profile before and after the correction was obtained, as shown in Fig. 14. 


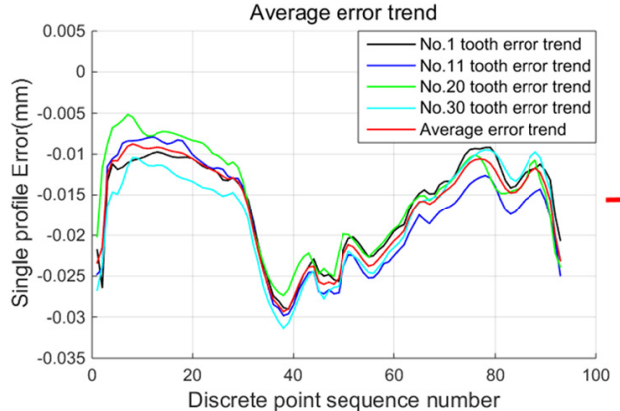

(a) Before correction

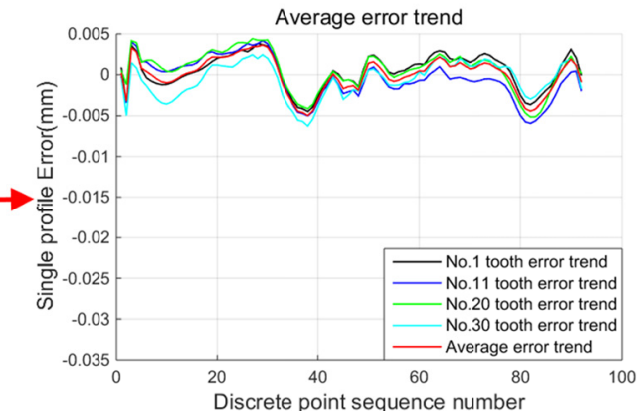

(b) After correction

Fig. 14 Average error change trend of cycloidal gear before and after correction.

It can be seen from Fig. 14 that the error of the corrected profile was significantly reduced. The corrected error fluctuation was reduced to within $\pm 0.005 \mathrm{~mm}$. The machine-tool settings for the second grinding should be the difference between the theoretical grinding settings and the correction values, as listed in Table 3. These grinding settings were fed back to the $\mathrm{CNC}$ gear grinding machine again, and the second grinding was completed.

Figure 15 shows the tooth profile error of the cycloidal gear after the second grinding. It can be seen that the error fluctuation range has also reached $\pm 0.005 \mathrm{~mm}$, which is basically consistent with the correction results in Fig. 14 . Obviously, after only one quantitative correction, the tooth profile error has been significantly reduced. Although there are still slight differences in the error values, the consistency of the results of the correction and grinding and the significant reduction of the error illustrated the correctness and effectiveness of the quantitative correction method.

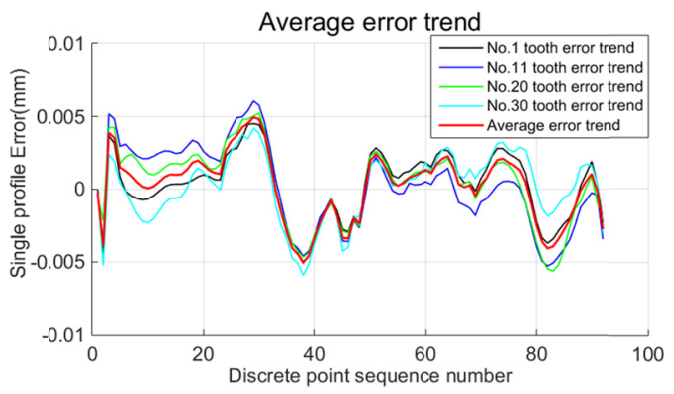

Fig. 15 Average error trend after second grinding.

Furthermore, Figure 16 shows a comparison of the total error trend of the cycloidal gear before and after the grinding. It can clearly be seen that after only one quantitative correction, the profile error curve of the cycloidal gear has fluctuated near the zero line, and the error value has also been greatly reduced.

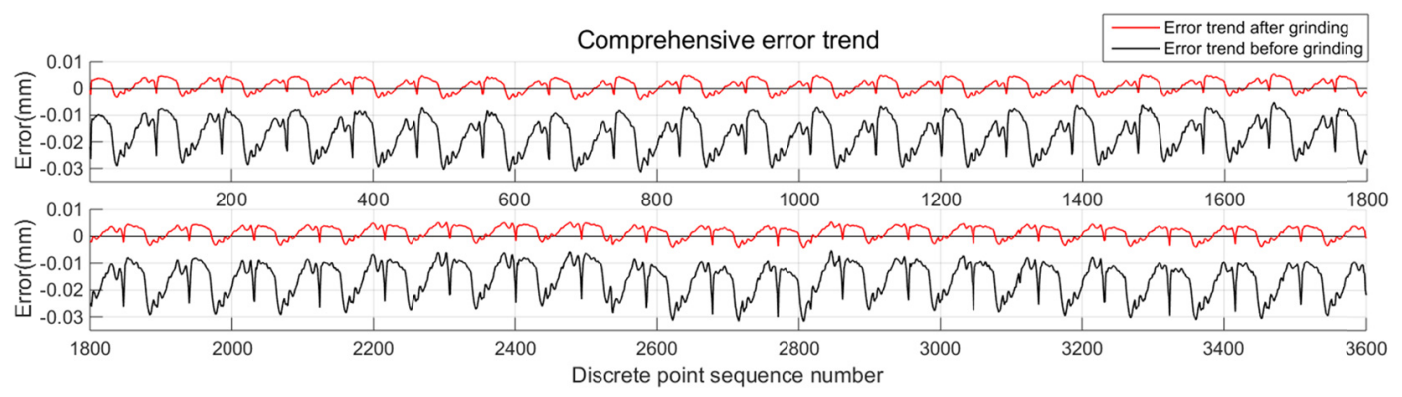

Fig. 16 Error fluctuation of cycloidal gear before and after grinding process.

In addition, the results of a statistical analysis of the error variation of the cycloidal profile before and after the grinding are listed in Table 4. 
Tianxing Li, Jinfan Li, Deng, Tian and Yulong Li, Journal of Advanced Mechanical Design, Systems, and Manufacturing, Vol.14, No.4 (2020)

Table 4 Error statistics of cycloidal gear before and after grinding (unit: $\mu \mathrm{m}$ ).

\begin{tabular}{ccccccc}
\hline & Error item & $\begin{array}{c}\text { Maximum } \\
\text { value }\end{array}$ & $\begin{array}{c}\text { Minimum } \\
\text { value }\end{array}$ & $\begin{array}{c}\text { Average } \\
\text { value }\end{array}$ & $\begin{array}{c}\text { Comprehensive } \\
\text { tolerance } F_{n}\end{array}$ & $\begin{array}{c}\text { Objective } \\
\text { function }\end{array}$ \\
\hline First & Single profile error & -8.4 & -29.8 & -16.9 & 21.4 & 29917 \\
grinding & Full profile error & -5.4 & -31.7 & -18.5 & 26.3 & 11113256 \\
\hline Second & Single profile error & 5.0 & -5.5 & 0.5 & 10.5 & 578 \\
grinding & Full profile error & 7.7 & -8.8 & 0.6 & 16.5 & 20822 \\
\hline
\end{tabular}

The data comparison shows that after the correction and grinding, the full profile error and single profile error of the cycloidal gear have been effectively reduced. The corrected objective function value shows a rapid downward trend, with a decrease from $11113256 \mu \mathrm{m}^{2}$ to $20822 \mu \mathrm{m}^{2}$. The values for the comprehensive tolerance, $F_{n}$, of the single and full profiles were reduced by almost $50 \%$. The correction effect was very good. It can be concluded that the profile error could be effectively reduced using only one correction and two grinding operations, and the quantitative correction method proposed in this paper could improve the grinding accuracy of the cycloidal gear.

\section{Conclusions}

Controlling the grinding accuracy of a cycloidal gear is an important prerequisite to ensure that a precision reducer has good motion accuracy and a long service life. Based on the error data from a gear measuring center, this paper proposed a method to improve the grinding accuracy of the cycloidal gear of a precision reducer, which allows the quantitative correction of the grinding error based on mathematical analysis and reverse engineering. This method can quickly and accurately obtain correction values for the machine-tool settings based on the grinding error and effectively reduce the grinding error. It solves a technical problem of the traditional grinding method, which can only qualitatively and subjectively correct the error without providing quantitative control, and provides an effective way to control and improve the machining accuracy of the key parts of a precision reducer. The authors expect that the research method will play an important role in the process of the design, machining, measurement, and meshing performance analysis of cycloidal gears.

\section{Acknowledgements}

This research is supported by the National Natural Science Foundation of China (No. U1504522, 51705134, and 51775171), Key Scientific Research Projects of Higher Education Institutions in Henan Province of China (No. 20A460010) and Key Scientific and Technological Project in Henan Province of China (No. 202102210079).

\section{References}

Chang, S. L., Studies on epitrochoid gear for cycloid drives, Chinese Journal of Mechanics Series A (English Edition), Vol.19, No.2 (2003), pp.271-278.

Chen, T. T., Wang, J. P., Huang, G. M., Hsu, M. H., Chen, C. L., Hong, B. W. and Wey, J. M., High-precision technology with negative punch clearance for the manufacturing of a cycloid pump, International Journal of Advanced Manufacturing Technology, Vol.95, No.1-4 (2018), pp.1179-1183.

Han, L. S. and Guo, F., Global sensitivity analysis of transmission accuracy for RV-type cycloid-pin drive, Journal of Mechanical Science and Technology, Vol.30, No.3 (2016), pp.1225-1231.

Kawahara, S., Yoshioka, T., Ohishi, K., Hien, N., Miyazaki, T. and Yokokura, Y., Vibration suppression control method using extended state observer for angular transmission error in cycloid gear, IEEJ Transactions on Industry Applications, Vol.134, No.3 (2014), pp.241-251.

Lai, T. S., Design and machining of the epicycloid planet gear of cycloid drives, International Journal of Advanced Manufacturing Technology, Vol.28, No.7-8 (2006), pp.665-670.

Li, B., Du, J. W., Chen, L., Xu, H. J. and Hou, C. Y., Transmission error analysis for industrial robot RV reducer, Journal of Xi'an Jiaotong University, Vol.51, No.10 (2017), pp.1-6 and 46 (in Chinese).

Li, J. B., Zhang, P. Z., Feng, L. X., Yin, G. X., Chen, Z., Ma, W. S., Xu, A. J., Nie, S. W. and Zhang, H., Information 
description and integration of spiral bevel gear manufacturing process under networked manufacturing mode, Journal of the Brazilian Society of Mechanical Sciences and Engineering, Vol.41, No.5 (2019), pp.234.

Li, T. X., Li, J. B., Deng, X. Z., Yang, J. J., Li, G. G. and Ma, W. S., A new digitized reverse correction method for hypoid gears based on a one-dimensional probe, Measurement Science and Technology, Vol.28, No.12 (2017), pp.125004.

Li, T. X., Zhou, J. X., Deng, X. Z., Li, J. B., Xing, C. R., Su, J. X. and Wang, H. L., A manufacturing error measurement methodology for a rotary vector reducer cycloidal gear based on a gear measuring center, Measurement Science and Technology, Vol.29, No.7 (2018), pp.075006.

Li, X., Chen, B. K., Wang, Y. W. and Lim, T. C., Mesh stiffness calculation of cycloid-pin gear pair with tooth profile modification and eccentricity error, Journal of Central South University, Vol.25, No.7 (2018), pp.1717-1731.

Li, X., Li, C. Y., Wang, Y. W., Chen, B. K. and Lim, T. C., Analysis of a cycloid speed reducer considering tooth profile modification and clearance-fit output mechanism, Journal of Mechanical Design, Transactions of the ASME, Vol.139, No.3 (2017), pp.033303-1.

Lin, K. S., Chan, K. Y. and Lee, J. J., Kinematic error analysis and tolerance allocation of cycloidal gear reducers, Mechanism and Machine Theory, Vol.124, No.6 (2018), pp.73-91.

Litvin, F. L. and Feng, P. H., Computerized design and generation of cycloidal gearings, Mechanism and Machine Theory, Vol.31, No.7 (1996), pp.891-911.

Liu, C., Shi, W. K., Han, Z. H. and Xu, L., Machining method of composite cycloid gear based on the kinematic control of shaping cutter, Journal of South China University of Technology (Natural Science), Vol.46, No.2 (2018), pp.3137 (in Chinese).

Liu, Z. F., Zhang, T., Wang, Y. D., Yang, C. B. and Zhao, Y. S., Experimental studies on torsional stiffness of cycloid gear based on machining parameters of tooth surfaces, International Journal of Precision Engineering and Manufacturing, Vol.20, No.6 (2019), pp.1017-1025.

Tran, T. L., Pham, A. D. and Ahn, H. J., Lost motion analysis of one stage cycloid reducer considering tolerances, International Journal of Precision Engineering and Manufacturing, Vol.17, No.8 (2016), pp.1009-1016.

Wang, B., Fan, M. X., Sun, X., Li, J. B., Xu, A. J., Li, G. G. and Yang, J. J., Cutter position calculation of machining equal base circle bevel gears with a pot-shaped milling cutter, International Journal of Advanced Manufacturing Technology, Vol.87, No.9-12 (2016), pp.2625-2637.

Wang, P., Li, J. and Jin, Y. Q., A study on the design of slicing cutter for cycloid gear based on conjugate theory, International Journal of Advanced Manufacturing Technology, Vol.98, No.5-8 (2018), pp.2057-2068.

Wang, T. M. and Tao, Y., Research status and industrialization development strategy of Chinese industrial robot, Jixie Gongcheng Xuebao, Vol.50, No.9 (2014), pp.1-13 (in Chinese). 